\title{
Transplantation of mesenchymal stem cell sheet to treat acute hind-limb ischemia: A preclinical study
}

\author{
Thuan Minh Le ${ }^{1,2}$, Giang Thi Dang ${ }^{2,3}$, Huynh Duc Phat ${ }^{1,2}$, Vu Bich Ngoc $2,3, *$ (1)
}

${ }^{1}$ Labotatory of Stem Cell Research and Application, University of Science Ho Chi Minh City, Viet Nam

${ }^{2}$ Viet Nam National University, Ho Chi Minh City, Viet Nam

${ }^{3}$ Stem Cell Institute, University of Science Ho Chi Minh City, Viet Nam

\section{Correspondence}

Vu Bich Ngoc, Viet Nam National University, Ho Chi Minh City, Viet Nam

Stem Cell Institute, University of Science Ho Chi Minh City, Viet Nam

Email: ngocvu@sci.edu.vn; vbngoc@hcmus.edu.vn

\section{History}

- Received: April 04, 2021

- Accepted: Jun 20, 2021

- Published: Jun 30, 2021

DOI : 10.15419/bmrat.v8i6.677

\section{Check for updates}

\section{Copyright}

( ) Biomedpress. This is an openaccess article distributed under the terms of the Creative Commons Attribution 4.0 International license.

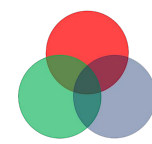

BiolMedPress

The Open Access Publisher

\begin{abstract}
Introduction: Mesenchymal stem cell (MSC) transplantation has been reported as a promising therapy for acute limb ischemia (ALI). However, the treatment efficacy is limited to only certain improvements. Therefore, this study aims to improve the treatment efficacy of MSC transplantation through the use of MSC sheets produced from MSCs' cultured in fibrin scaffold (Fi-MSCs) in ALI models. Methods: MSCs were isolated and expanded from human umbilical cord tissue. The fibril scaffold was produced from human umbilical cord blood. Fi-MSCs were prepared by mixing MSCs with fibril according to a published protocol, and the Fi-MSC sheets were implanted directly into ligated and transected sites in the hind limbs of ischemic models (treatment group - group I). The results were compared with that of the control group (group II) in which mice were injected with saline. The treatment efficacy was recorded and evaluated through the following assays: limb morphology, $\mathrm{SpO}_{2}$, blood perfusion, angiogenesis, and histological morphology on days 7, 14, and 28 after treatment. Results: The results indicate that the transplantation of Fi-MSC sheets positively affected the acute ischemia hind-limb mouse models. On day 7 post-transplantation, the $\mathrm{SpO}_{2}$ index recorded at feet in group I (treatment) significantly increased from $79.24 \%+1.43 \%$ to $89.40 \%$ $+1.65 \%$ ( $p$-value $<0.05$ ), while in group II (control), the $\mathrm{SpO}_{2}$ index slightly increased from $76.52 \%$ $+1.63 \%$ to $77.00+1.15$ ( $p$-value $>0.05$ ). Besides, there were $60.00 \%$ (auto-recovery), $13.33 \%$, and $26.67 \%$ mice at damage grades $0, \mathrm{I}$, and II, respectively, in the control group compared to $80 \%$, $20 \%$, and $0 \%$ mice at damage grades 0,1 , and II, respectively. Moreover, in group I, all mice showed improved blood reperfusion, neovascular, and repaired muscle tissue compared to group II. Conclusion: Fi-MSC sheet transplantation positively reduced injury and improved blood perfusion ALI in the Swiss mouse model.
\end{abstract}

Key words: biological scaffold, fibrin, hind-limb ischemia, mesenchymal stem cells, neovascularization

\section{INTRODUCTION}

Peripheral artery disease (PAD) is commonly associated with atherosclerosis that limit blood supply to both the upper and lower extremities, often affecting the lower extremities more severely ${ }^{1}$. PAD is primarily prevalent in middle-aged people, with a rate of about $20 \%{ }^{1}$. Acute hind-limb ischemia is one of the diseases associated with PAD. Critical limb ischemia is described as a sudden decrease in blood flow to the limb. It can result in amputation or mortality if not treated appropriately ${ }^{2}$. Approximately $1-1.5$ individuals per 10,000 people develop acute limb ischemia (ALI) every year ${ }^{2}$. Therefore, extensive research is required to improve the effectiveness of treatment.

The causes of ALI can be divided into two groups: traumatic (5\%) and nontraumatic (95\%). The causes of nontraumatic ALI include arterial embolism (30\%), plaque-induced arterial thrombosis (40\%), popliteal aneurysm thrombosis (5\%), and graft thrombosis $(20 \%)^{3,4}$.
Symptoms of ALI develop rapidly within several minutes, hours, or days after occlusion. The disease progression of a patient's ALI is described by the term "6Ps": pain, pallor, paralysis, pulse deficit, paresthesia, and poikilothermia ${ }^{3}$. Additionally, patients experience intermittent pain to pain at rest, paresthesia, muscle weakness, paralysis, and even gangrene.

Most of the therapies used for ALI are based on thrombolysis, such as streptokinase, saline jets, ultrasound waves, surgical treatment, or intravascular therapy ${ }^{5}$. Both saline jets and ultrasound waves are used to lyse blood clots. Saline jets can dislodge the blood clot through the Bernoulli effect, while ultrasonic waves can cause physical fragmentation of the blood $\operatorname{clot}^{4}$. Surgical revascularization is administered immediately to eliminate ischemia as soon as possible in patients identified with the severity classification. Intravascular therapy is an initial therapy option for a low level because they have more treatment time ${ }^{2}$. 
In the recent years, stem cell therapy has been considered a promising approach for the treatment of ALI. It shows significant potential in the treatment of ischemia by angiogenesis at ischemic sites ${ }^{1}$.

Angiogenesis has become a new focus for the treatment of limb ischemia. Angiogenesis reestablishes blood and nutrients supply to the ischemic area, reducing ischemic severity ${ }^{6,7}$. Proangiogenic cytokines such as vascular endothelial growth factor (VEGF) and basic fibroblast growth factor (bFGF) are crucial in promoting blood vessel formation and increase collateral circulation in ischemia patients ${ }^{5}$. Moreover, hUC-MSCs are also known to secrete large amount of cytokines because of the growth factors (GFs) such as VEGF and FGF-2. hUC-MSCs have been proven to promote angiogenesis in vivo and in vitro and have high expression levels of VEGF- $1 \alpha$, MMP-9, VEGFR, and HIF- $1 \alpha^{8}$. To summarize, UCMSCs contribute significantly to the regeneration of blood vessels and reduce the symptoms of ischemic limb ${ }^{8}$.

Fibrin is an important component responsible for homeostasis and is used as a biopolymer scaffold in tissue engineering. Fibrin also has a key role in angiogenesis ${ }^{9}$. During wound healing, fibrin provides a suitable environment for angiogenesis. Therefore, the blood supply is restored ${ }^{10,11}$. The structure and characteristics of fibrin can trigger the formation of new blood vessels in the wound. Biodegradable substrates (matrix) such as fibrin are considered ideal for tissue engineering applications because they can enable complete tissue regeneration and leave no trace of the original scaffold. The fibrin matrix has widely been used to research blood vessels for tissue engineering and the treatment of ischemic disease. Fibrin's flexibility in supporting angiogenesis has led it to become an ideal substrate for researching angiogenesis in the body $^{12}$.

Therefore, this study aims to evaluate the treatment efficacy of mesenchymal stem cell (MSC) sheet transplantation from umbilical-cord-derived MSCs and fibril scaffolds in acute hind-limb ischemic mouse models.

\section{METHODS}

\section{Animals and samples}

In this study, all procedures and manipulations on animals were approved by the Animal Welfare Committee of the Stem Cell Institute, University of Science, VNU-HCM, Vietnam. Swiss six-month-old, male healthy mice were used to establish the acute hind-limb ischemic models.

Umbilical cord tissues and blood samples were collected at hospitals after birth with consent forms.
These samples were used for research with the approval of mothers and the hospital ethical committee (District 2, Ho Chi Minh City, VN). All samples were screened for HIV-1/2, HBV, HCV, and CMV. Only samples negative with all these viruses were used in the study.

\section{Culture and expansion of MSCs}

MSCs from the human umbilical cord were isolated as per the published protocols ${ }^{13-15}$. Briefly, the umbilical cord tissues were collected from the donor with the consent form and the approval of the hospital ethical committee (District 2 General Hospital, Ho Chi Minh City, VN). The tissues were washed twice with a washing buffer solution added with antibioticmycotic. Then they were dissected into small fragments $\left(1-2 \mathrm{~mm}^{2}\right)$ and placed into the T-75 flasks with $5 \mathrm{~mL}$ of MSCCult I primary medium. These flasks were incubated at $37^{\circ} \mathrm{C}$ with $5 \% \mathrm{CO}_{2}$ for 5 days. When the migrated cells from the tissues got $70 \%$ confluence, they were subcultured to new flasks and discarded tissues. The MSC candidates were expanded and subcultured to the third passage using the MSCCult I medium before they were characterized as MSCs according to the minimal criteria suggested by ISCT $^{16}$. All chemicals and media were bought from Regemedlab (Ho Chi Minh City, VN).

\section{Characterization of MSC candidates}

MSC candidates were characterized as MSCs based on the minimal criteria for MSCs suggested by ISCT in $2006^{16}$. The expression of MSC-specific marker profile with CD14, CD19, CD34, CD45, CD73, CD90, CD105, and HLA-DR was evaluated. The cells were washed twice with washing buffer (Regenmedlab, Ho Chi Minh City, VN). Subsequently, the cells were stained with the antibodies and incubated at room temperature for 15 minutes in the dark. Then, the cells were washed twice with washing buffer. The stained cells were analyzed using FACSCalibur and BD CellQuest ${ }^{\mathrm{TM}}$ Pro software systems (BD Biosciences, Franklin Lakes, NJ).

For in-vitro differentiation assays, the cells were seeded in a 4 -well plate with a density of $10^{4}$ cells $/ \mathrm{mL}$ with $100 \mu \mathrm{L}$ of MSCCult I medium for adherence in 48 hours. Then, the media were replaced with suitable differentiation-inducing media for adipogenesis, osteogenesis, and chondrogenesis (bought from Thermo Fisher) for adipocyte, osteocyte, and chondrocyte differentiation, respectively. The cells were cultured for 14 days in the adipogenic medium, 21 days in the chondrogenic medium, and 28 days in the 
osteogenic medium. The media were refreshed every four days. Finally, the cells were stained with Oil Red (Sigma-Aldrich, USA), Alcian Blue (Sigma-Aldrich, USA), and Alizarin Red (Sigma-Aldrich, USA) to determine their ability to differentiate into adipocytes, chondrocytes, and osteocytes, respectively.

\section{MSC sheet production}

MSC sheets (Fi-MSCs) were created by merging MSCs in the fibrin scaffold. The fibrin scaffold was prepared according to a commercial kit's instruction (5PRP, version U, Regenmedlab, Ho Chi Minh City, $\mathrm{VN})$. Briefly, the umbilical cord blood was transferred to a 50-mL 5PRP tube and centrifuged at 3,000 RPM for 10 minutes. One-third of the upper plasma was removed, and two-third of the lower (platelet-rich plasma - PRP) was collected in a new $50-\mathrm{mL}$ centrifuge tube. Then, $10^{6}$ MSCs were suspended in 0.5 $\mathrm{mL}$ of PRP. The suspension of MSCs and PRP was loaded into a 4-well plate with $0.5 \mathrm{~mL}$ per well. Next, $0.12 \mathrm{~mL}$ of $0.8 \mathrm{M} \mathrm{CaCl}_{2}$ solution was added to each well.

\section{Acute hind-limb ischemic mouse modeling and Fi-MSC transplantation}

Acute hind-limb ischemic mice were used according to the published protocols using 6-month-old healthy mice $^{17-19}$. The mice were anesthetized using xylazine (4 mg/kg) and Zoletil ( $5 \mathrm{mg} / \mathrm{kg})$. Femoral hair was shaved, and then an incision of approximately 1.5 $\mathrm{cm}$ long was made in the direction of the femoral blood vessel to open femoral skin at thigh and display femoral veins and arteries.

Both veins and arteries were ligated at two positions at the lateral circumflex artery branches and distally near the popliteal and saphenous artery. The vessels were then cut out at middle of the two ligations. For the treatment group (group I), an Fi-MSC sheet was implanted at the cut site. For the control group, saline was used to wash the cut site. Finally, the skin was stitched, and the wound was coated with povidoneiodine.

\section{Regeneration evaluation of damaged limbs}

The degree of ischemic damage at the surgery was recorded, evaluated, and classified according to the guidelines of Goto et al. (2006) ${ }^{20}$ as follows: grade 0 , normal limb without swelling, necrosis, or atrophy of muscle; grade I, necrosis limited to toes; grade II, necrosis extending to the foot; grade III, necrosis extending to the knee; and grade IV: necrosis extending to the femoral region.
Blood flow was assessed indirectly by monitoring the change in oxygen saturation in the blood. This change was detected by the sensor set point in the $\mathrm{SpO}_{2}$ measuring device (Contec 08A, China). Furthermore, blood flow to the hind limbs was also evaluated using trypan blue assay. The $0.04 \%$ trypan blue solution was directly injected into the tail vein of the mice. The staining time of the dye that went to the hind limb was recorded based on the blue staining of toes observed by naked eyes.

The formation of new blood vessels was evaluated at 7 days, 14 days, and 28 days after operation. The mice were fixed to the surgical chamber, and the femoral muscle area was assessed. The formation of new blood vessels was observed directly under a stereomicroscope (Zeiss, Germany).

Histological analysis of the limb tissues was also performed at 7 days, 14 days, and 28 days after occlusion. Muscle tissue in hind limbs was biopsied and fixed in 4\% PFA for 24 hours. After fixing in PFA, the sample was fixed in a sucrose solution of $30 \%$ at $4^{\circ} \mathrm{C}$ and until slicing. The samples were cut and stained with hematoxylin and eosin dyes.

\section{Statistical analysis}

In this study, VesSeg_V0.1.4 software was used to quantify blood vessels. Data were analyzed by the software GraphPad Prism 8. Statistical analysis was performed by t-test. P-value $<0.05$ was considered significant. All data were presented as mean \pm standard deviation.

Light microscope image ofblood vessels before occlusion (A), immediately incision (B); 7 days (D, G), 14days (E, H), and 28 days (F,I) after occlusion of the femoral artery. Measurement ofnew blood vessels formed at 28 days postoperative by using VesSeg_V0.1.4software $\left(\mathrm{C},{ }^{\star * \star} \mathrm{p}<0.0001\right)$.

\section{RESULTS}

\section{Isolation and characterization of human umbilical cord-derived mesenchymal stem cells}

Isolated MSCs adhered to the flask surface and displayed spindle-shaped fibroblastic-like morphology (Figure 1A). In vitro, the MSC candidates successfully differentiated into osteoblasts (Figure 1B) that were positive with Alizarin Red staining assay, chondroblasts (Figure 1C) positive with Alcian Blue, and adipocytes (Figure $1 \mathbf{D}$ ) positive with Oil Red.

The MSC candidates expressed all MSC-specific maker profiles suggested by ISCT. Indeed, they were positive with CD73 (99.4\%), CD90 (99.07\%), and 

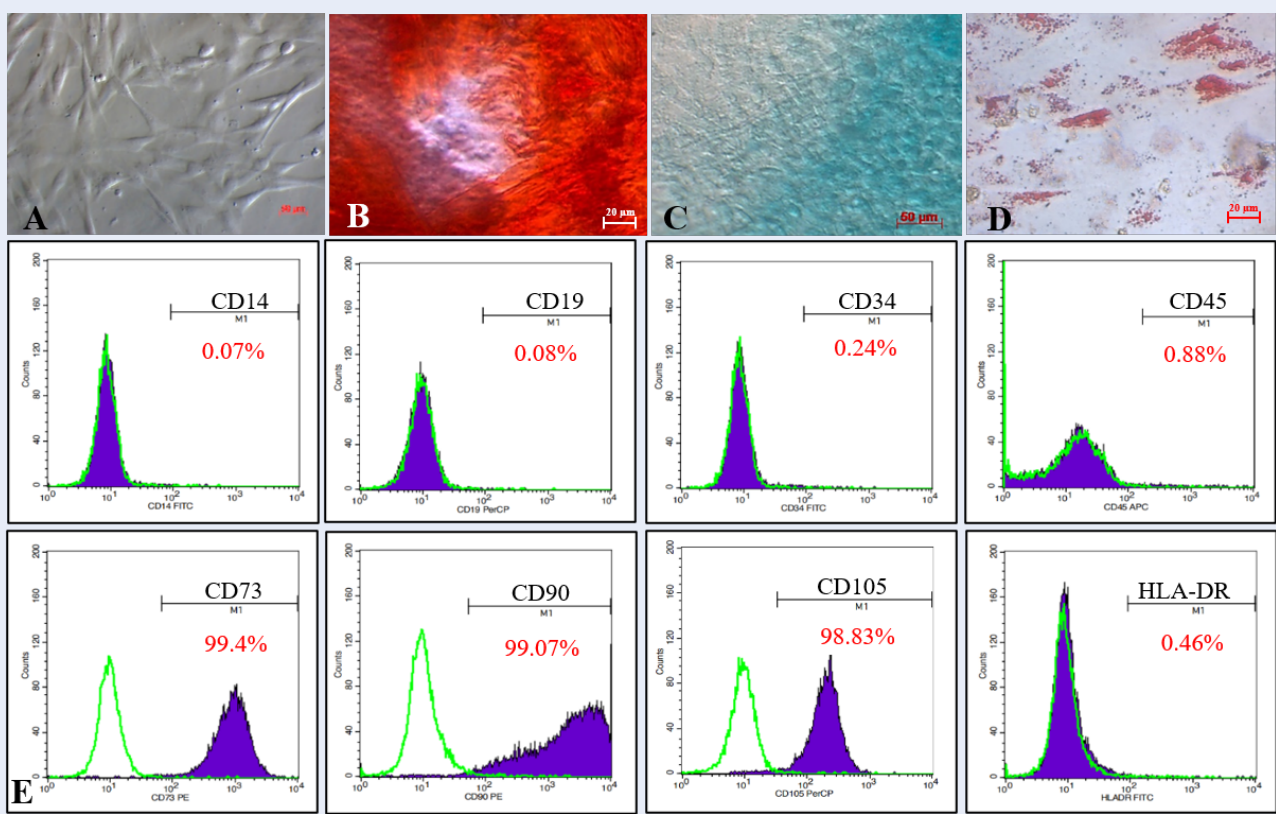

Figure 1: Isolated MSCs from the human umbilical cord displayed the MSC phenotype suggested by ISCT. Isolated cells displayed the fibroblast shape when adhered to the flask surface $(\mathbf{A})$. Induced cells were positive with Alizarin red, Acian blue, and Oil red $\mathrm{O}$ dyes, respectively, for differentiating into osteoblasts, chondroblasts, andadipocytes (B, C, D). Cells were strongly expressed CD73 (99.4\%),CD90 (99.07\%), and CD105 (98.83\%); while negative with CD14 (0.07\%), CD19 (0.08\%), CD34 (0.24\%), CD45 (0.88\%)and HLA-DR (0.46\%)(E).
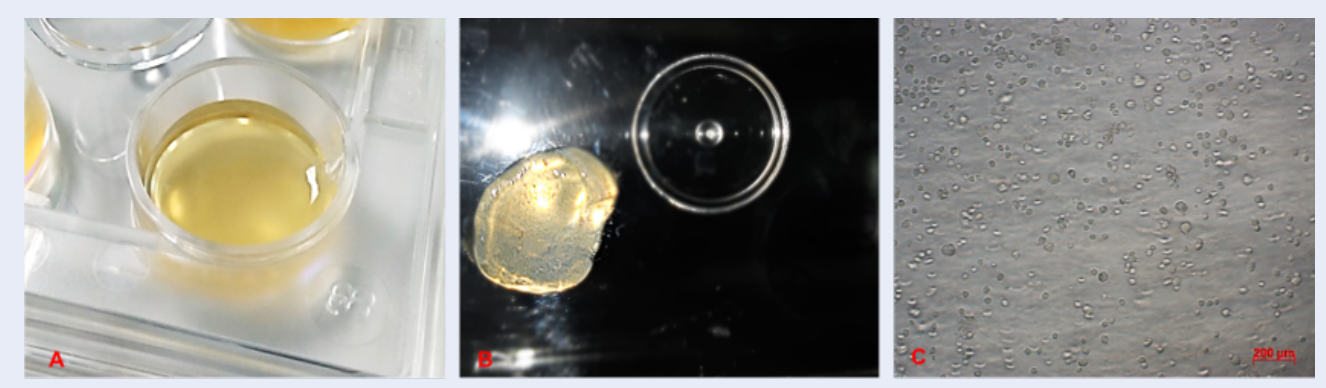

Figure 2: Fi-MSC sheets formed from mixing MSCs in PRP solution then activated by $\mathrm{CaCl}_{2}$. Fi-MSC formed a 4-well culture plate (A), and after picking out the culture plate (B), and MSCs were distributed in the fibril scaffold (C).

CD105 (98.83\%), and negative with CD14 (0.07\%), CD19 (0.08\%), CD34 (0.24\%), CD45 (0.88\%), and HLA-DR (0.46\%; Figure 1 E).

\section{Production of Fi-MSC sheets}

Fi-MSCs were formed as semi-solid after being activated with $\mathrm{CaCl}_{2}$ in 2 minutes on a well culture plate (Figure 2A-B). These cells were evenly distributed on and in a fibrin scaffold with spherical morphology Figure $2 \mathrm{C}$ ).
Treatment efficacy of Fi-MSC sheets on acute hind-limb ischemia mice models Limb oxyhemoglobin saturation-SpO

At 30 minutes after occlusion, limb oxygen saturation in mice from both control and treatment groups were significantly dereased compared to normal mice (without surgery of produce models; $\mathrm{p}<0.05$ ). Oxyen saturation levels recorded at limbs were $76.53 \%+$ $1.63 \%$ in the control group mice and $79.24 \%+0.79 \%$ in treatment group mice compared with $95.67 \%+$ $0.29 \%$ in normal mice. From day 7 to day 14 , the $\mathrm{SpO}_{2}$ 


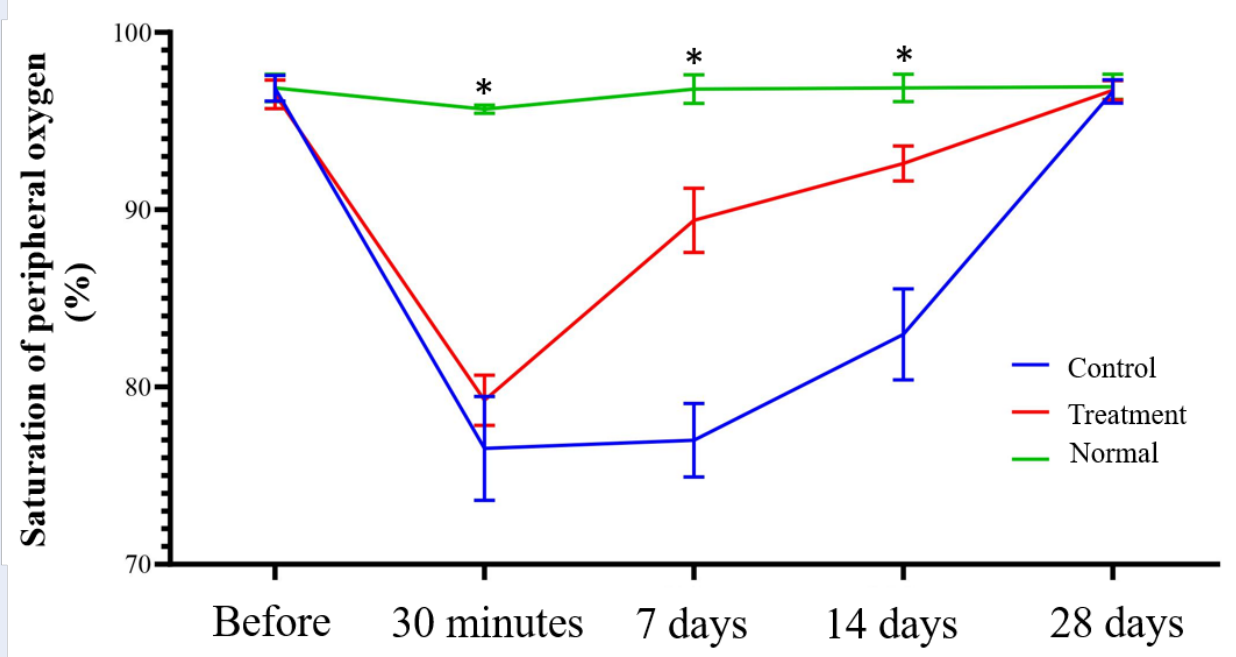

Times

Figure 3: The changes of hemoglobin oxygen saturation in murine limbs at different times. The $\mathrm{spO}_{2}$ significantly after $30 \mathrm{~min}$ of ligations of blood vessels. In the treated mice with $\mathrm{MSC}$ sheets, the $\mathrm{SpO}_{2}$ increased dramatically after 7 and 14 days post-transplantation. While in control mice, after seven days, the $\mathrm{SpO}_{2}$ also gradually raised in auto-recovered mice.

index recorded at limbs in the control group mice slightly increased from $77.00 \%+1.15 \%$ to $82.97 \%$ $+1.84 \%$ and significantly increased from $89.40 \%$ $+1.00 \%$ to $92.60 \%+0.71 \%$ in treatment group mice $(\mathrm{p}<0.05)$. After 28 days, oxygen saturation recorded in the limbs of both treated and control mice that acquired auto-recovery returned to the normal level $(96.93 \%+0.88 \%, 96.67 \%+0.96 \%, 96.73 \%+0.67 \%$, respectively, for normal mice, treated mice, and autorecovered control mice) (Figure 3).

\section{Blood flow to the feet}

At 7 days after femoral occlusion, it took $185+10 \mathrm{~s}$ in treated mice (Figure 4A and 4D) compared with $490+127 \mathrm{~s}$ in control mice (Figure 4B and 4D) to for the trypan blue to reach from the tail vein to limb ( $p$ $<0.05$ ) and significantly longer in normal mice 77.33 $+1.78 \mathrm{~s}(\mathrm{p}<0.05$, Figure $4 \mathrm{C}$ and 4D).

\section{New blood vessel formation}

After 7 days of occlusion, new, small blood vessels appeared at the ligation sites (Figure 5D\&G). After 14 days of treatment, more and larger blood vessels were formed than in the control group (Figure 5E\&H). Moreover, a measurement of blood vessels using VesSeg_V0.1.4 software revealed that the total area of new vessels per field in the treatment group was
$11.41 \%+0.24 \%$ (Figure $5 \mathrm{~F} \& \mathrm{I}$ ) on day 28 , which is also larger than that of the control group with $6.76 \%$ $+0.59 \%$ (Figure 5C).

\section{Histological assessment}

At 7 days post transplantation, the H\&E staining of the muscle tissues at the thighs of mice in both control and treatment groups showed cytoplasmic fragmentation (Figure 6A and 6D). At 14 days, some new blood vessels, the usual morphology of cells, and muscle fibers were observed in the muscle tissues of treated mice (Figure 6E). Conversely, the muscle tissue structure collected from the control group mice contained some lesions in the cytoplasm and had lost muscle bundle (Figure 6B). After 28 days, the muscle tissue structure of both groups (auto-recovered control mice) were similar to the normal structure observed in the normal mice (Figure $6 \mathrm{C}$ and F). However, the muscle bundle structure in the treatment group seemed closer to that in normal mice (Figure 5 G).

\section{Hind-limb morphology and necrosis grade of mice}

At 3 days after surgery, there were significant difference in the percentage of mice with lesions at different grades, including grade 0 (Figure 7A), grade 


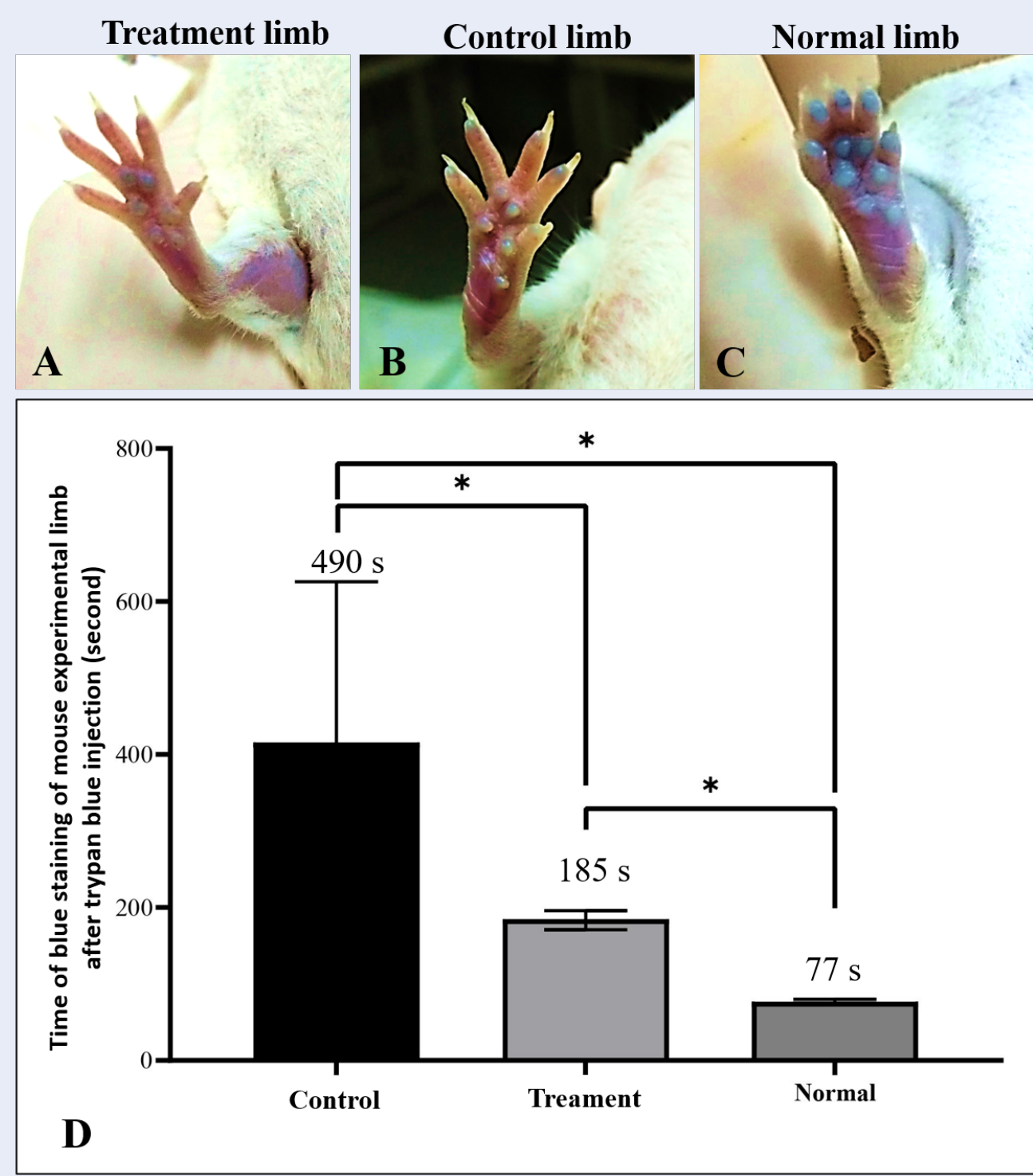

Figure 4: Measurement of the blood flows from vein tail to feet mice after seven days. Trypan blue Dye stained limb fads of treated (A), control (B) and normal (C) mice. The time that trypan blue could be stained feet pads were clearly different between groups (D) $\left({ }^{*} P<0.05, n=15\right)$.

I (Figure 7B), and grade II (Figure 7C) in both control and treatment group. In the control group, there were $46.6 \%, 33.30 \%$, and $20 \%$ mice with limb damage at grades $0, \mathrm{I}$, and II, respectively compared to $73.33 \%$, $26.27 \%$, and $0 \%$ of mice with limb damage grades 0 , I, and II respectively in the treatment group (Figure 7 D).

On day 7 post transplantation, there were $60.00 \%$ (auto-recovery), $13.33 \%$, and $26.67 \%$ mice at damage grades 0 , I, and II, respectively, in the control group compared to $80 \%, 20 \%$, and $0 \%$ mice at damage grades 0 , I, and II, respectively (Figure 7 D).

\section{DISCUSSION}

Acute limb ischemia is one of the most serious peripheral artery diseases. It can lead to amputation or even death. Traditional therapies have many limitations and do not stimulate new blood vessel formation. In the recent years, the umbilical cord-derived mesenchymal stem cells have emerged as a potential raw material for regenerative medicine therapy and showed the effectiveness of angiogenesis and injury recovery. 


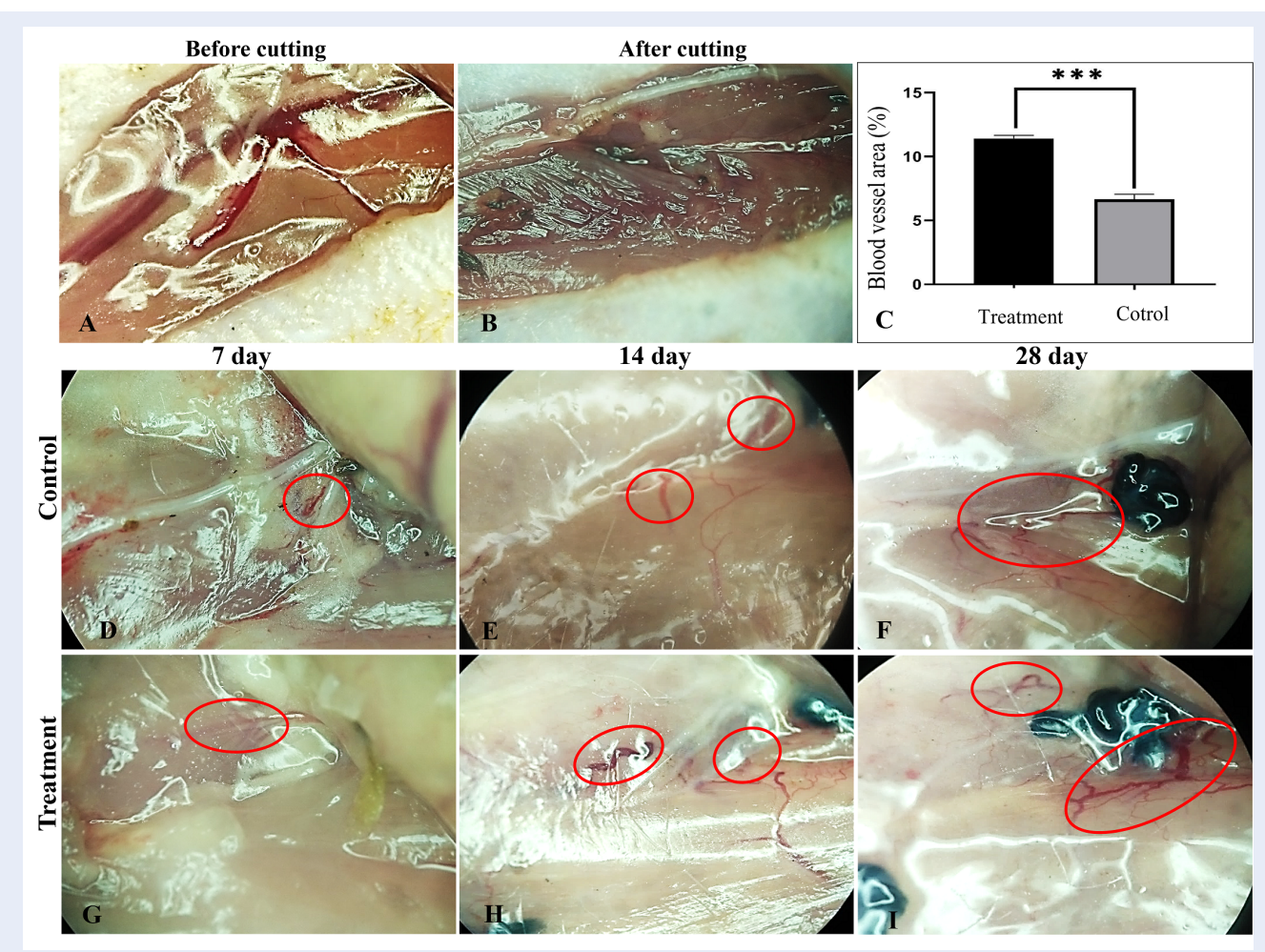

Figure 5: Light microscope image of blood vessels before occlusion (A), immediately incision (B); 7 days (D, G), 14 days $(E, H)$, and 28 days $(F, I)$ after occlusion of the femoral artery. Measurement of new blood vessels formed at 28 days postoperative by using VesSeg_V0.1.4software (C). ${ }^{* *} p<0.0001$

MSCs can produce GFs such as hepatocyte growth factor (HGF), fibroblast growth factor (FGF), VEGF, and high expression of factors that can regulate hematopoietic cell function such as CXCL12, vascular cell adhesion molecules 1, interleukin-7, angiopoietin-1 (Ang-1), and osteopontin ${ }^{21}$. These factors are essential to blood vessel formation. Moreover, MSCs also can promote the proliferation and migration of endothelial cells in injured tissue ${ }^{22}$ and promote angiogenesis through the production of vascular factors in ischemic tissues ${ }^{23}$. Clinically, Fan et al. (2011) demonstrated that MSC transplantation could promote vascular regeneration and improve and restore blood flow of diabetes patients with lower limb ischemia ${ }^{24}$.

In this study, isolated MSCs were confirmed as MSCs according to the standard recommended by Dominici et al. ${ }^{16}$. Moreover, the result showed that MSCs exhibited MSC phenotypes. This result is consistent with published studies ${ }^{14,25}$

The fibrin scaffold used in this study was derived from human umbilical cord blood. The fibrin scaffold contributes to the stimulation of new vascular formation and can support cells to proliferate, differentiate, and secrete cytokines by creating a three-dimensional environment $^{26}$ so they can release PDGF, TGF- $\beta 1$, EGF, VEGF, bFGF, and so on ${ }^{27}$. Moreover, the fibrin scaffold is a highly compatible biological substrate and contains many biological factors that are crucial in angiogenesis. This material is also well suited as a scaffold for the fabrication of new tissues. Therefore, the combination of fibrin and MSC creates a promising new material for ALI. This scaffold will help immobilize the stem cells at the desired location.

Although the femoral artery was excised, the most severe degree of necrosis of the mouse limb was amputation to the knee (grade II) according to the classification of Goto et al. ${ }^{20}$. The cutting of the femoral blood vessel caused a major disruption of blood flow to the underlying tissues such that trypan blue dye reached the walking pads slower than in normal mice, as when blood flow to the limbs decreases, the oxygen carried by the blood also decreases and A significant reduction in blood flow occurs within 1 hour of vascular ablation. However, the collateral system may be activated to reestablish perfusion. After 48 hours, reperfusion to the extremities was enhanced, and the rate 


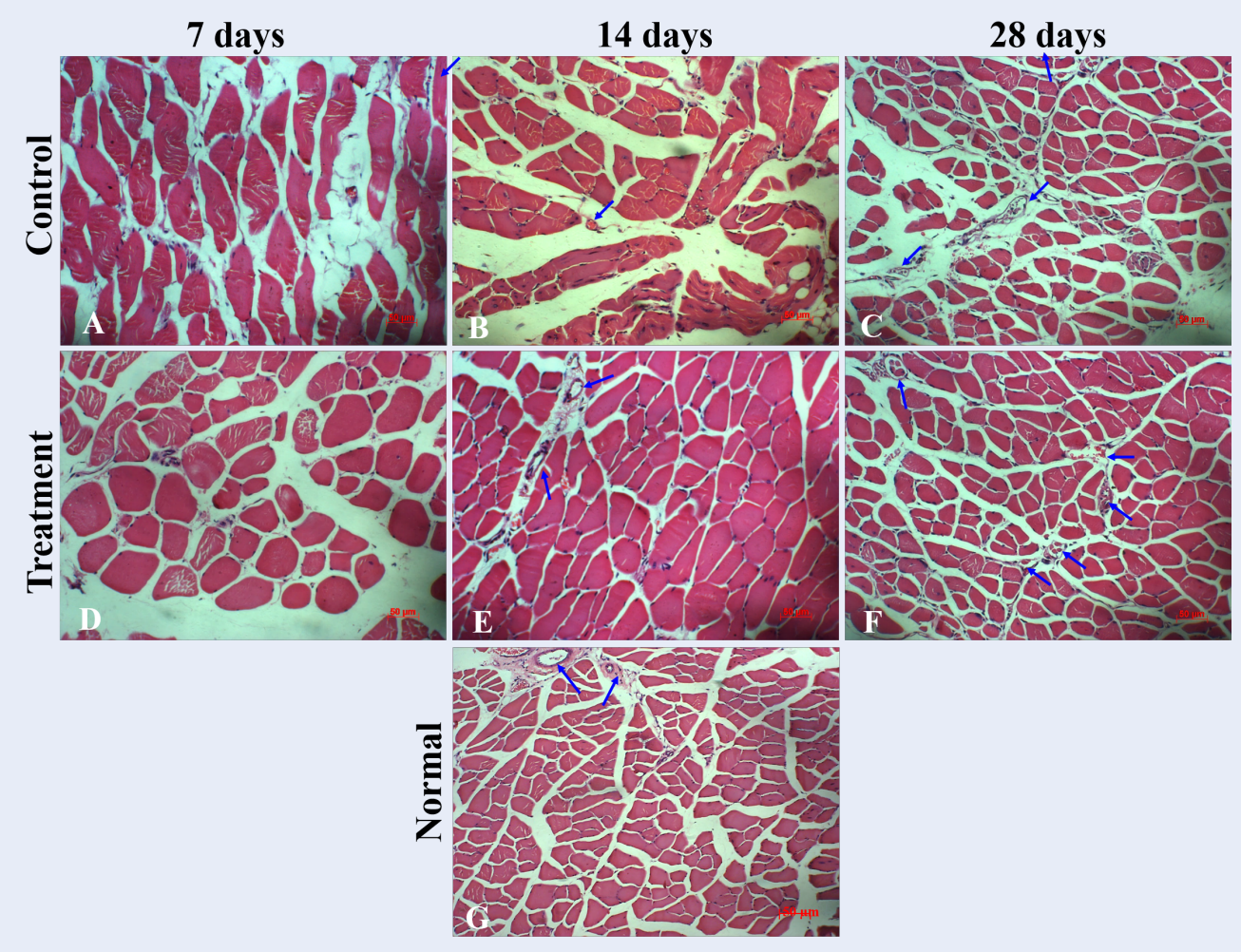

Figure 6: Histology structure analysis. Structure of muscle tissue stained with Hematoxylin and Eosin in nontreated groups (A-C), treated group(D-F), and normal (G) at day $7(\mathbf{A}, \mathrm{D})$, day $14(\mathbf{B}, \mathbf{E})$, and day $28(\mathbf{C}, \mathrm{F})$ after occlusion (green arrow show blood vessels)

of blood transport to the hind limbs improved significantly. This improvement was evident on day $7^{28}$. After 7 days at the vascular extremity, the increase of blood oxygen saturation was similar to that of a previously published study ${ }^{18}$.

The reduction of oxygen concentration in the blood flow causes a decrease in nutrition supply, thereby causing severe damage to the structure and function of the cells. Moreover, the disruption of blood circulation also leads to the accumulation of cellular waste products at extremely high concentrations, which is toxic to cells. This lead to cells being unable to repair their damage and enter the process of death instead. This can be one of the leading causes of tissue necro$\operatorname{sis}^{29}$.

Fi-MSC sheet transplantation on ischemic hind limb showed significant effect on preventing necrosis of hind limbs. The results showed that transplanted mice had significantly reduced tissue necrosis, quickly recovered their $\mathrm{SpO}_{2}$ index, sped up the blood circulation, and stabilized muscle tissue structure. Although, under hypoxia conditions, endogenous stem cells could be activated to proliferate and could differentiate into functional cells, such as smooth muscle cells or endothelial cells to form blood vessels this accelerate the wound repair process and reduces necrosis. In this case, transplanted MSCs could significantly contribute to this process. They can produce and secrete GFs to attract intrinsic stem cells to repair tissues, thereby accelerating wound healing ${ }^{30,31}$. Factors secreted by MSCs may include exosomes with signaling roles for endothelial cell recruitment and proliferation, promoting early events of angiogenesis, and thereby enhancing reperfusion at the injured area $^{22}$. MSCs also can secrete GFs such as HGF, FGF, VEGF, and high expression of hematopoietic regulatory factors such as CXCL12, vascular cell adhesion molecule 1, interleukin-7, and Ang- ${ }^{16}$, or some cytokines such VEGF-1 $\alpha$, MMP-9, VEGFR, and HIF$1 \alpha$ it $^{8}$. These are essential factors that promote angiogenesis in vivo and in vitro.

\section{CONCLUSIONS}

The study primarily showed that Fi-MSC sheets helped effectively treat acute ischemic hind limb in 


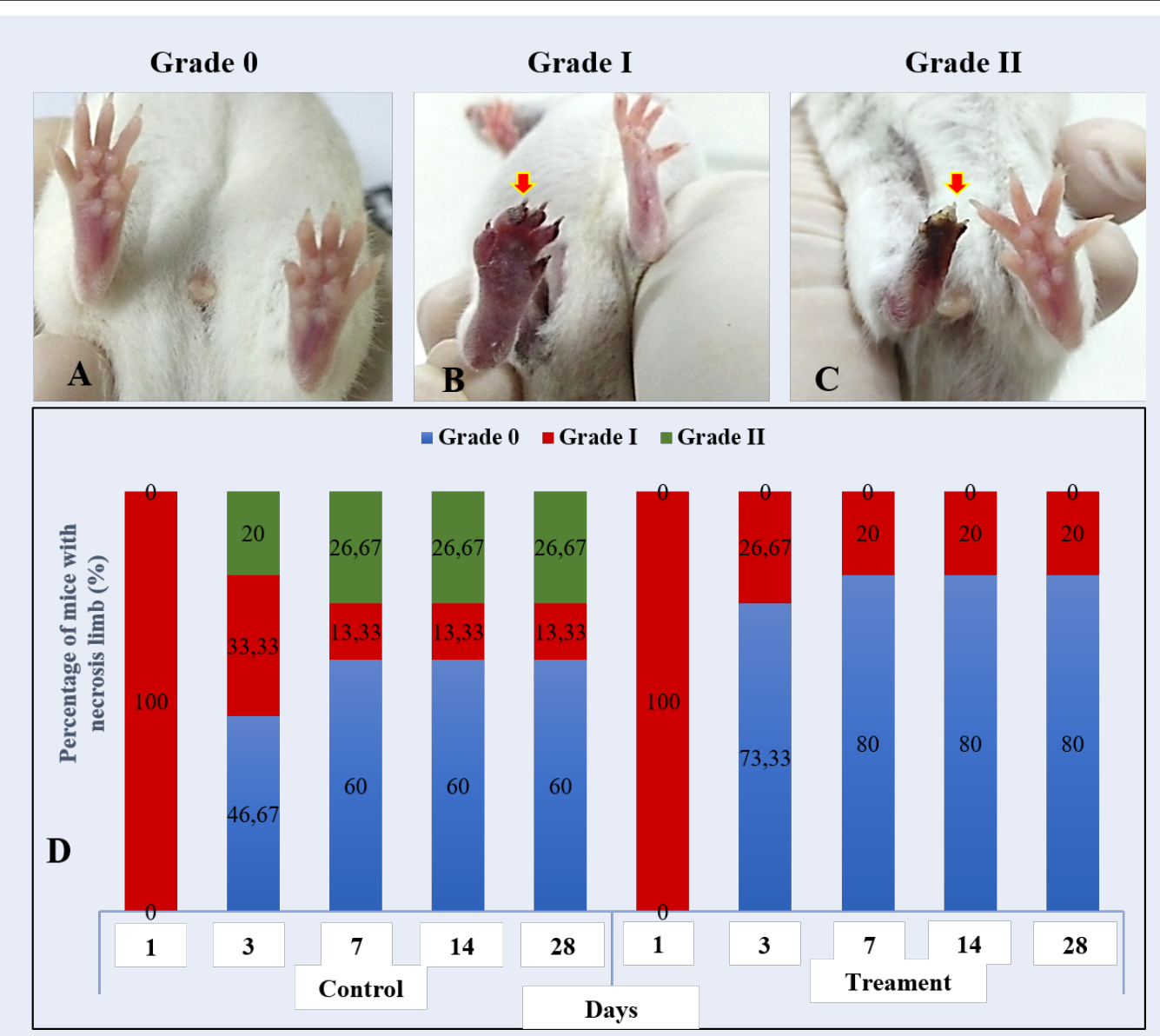

Figure 7: Recovery of murine hind limb ischemia in different groups. Muscle damages were classified as grade 0, I, II (Grade 0: normal limb, without swelling, necrosis, or atrophy of muscle (A); grade I: necrosis limiting to toes; and grade II: necrosis extending to the foot (B); grade III, necrosis extending to the knee; grade IV: necrosis extending to a femoral (C)). Evaluation ischemia score at day 1, 3, 7, 14, and 28 (D).

murine models. The MSCs from umbilical cord tissues may significantly contribute to neoangiogenesis in combination with fibril effects. Furthermore, the transplantation of Fi-MSCs significantly reduced tissue necrosis and stimulated the tissue regeneration of injured tissues. These results indicate a new promising therapy to treat acute ischemia. However, further studies with more mice to evaluate the side effects of this therapy are required and the existence of MSCs before this study should be translated to clinical trials.

\section{ABBREVIATIONS}

ALI: Acute limb ischemia

Fi-MSCs: mesenchymal stem cells seeded- fibrin scaffold

HLA-DR: Human Leukocyte Antigen - antigen D Related
hUC-MSCs: human umbilical cord mesenchymal stem cells

PAD: Peripheral artery disease

SD: Standard Deviation

PRP: Platelet Rich Plasma

$\mathrm{SpO}_{2}$ : Saturation of peripheral oxygen

VEGF: fibroblast growth factor

\section{ACKNOWLEDGMENTS}

This research is funded by University of Science, VNU-HCM under grant number T2020-30.

\section{AUTHOR'S CONTRIBUTIONS}

All authors equally contributed in this work. All authors read and approved the final version of the manuscript for submission. 


\section{FUNDING}

University of Science, VNU-HCM under grant number T2020-30.

\section{AVAILABILITY OF DATA AND MATERIALS}

Data and materials used and/or analyzed during the current study are available from the corresponding author on reasonable request.

\section{ETHICS APPROVAL AND CONSENT TO PARTICIPATE}

Not applicable.

\section{CONSENT FOR PUBLICATION}

Not applicable.

\section{COMPETING INTERESTS}

The authors declare that they have no competing interests.

\section{REFERENCES}

1. Jeong IH, Bae WY, Choi JS, Jeong JW. Ischemia induces autophagy of endothelial cells and stimulates angiogenic effects in a hindlimb ischemia mouse model. Cell Death Dis. 2020;11(8):624. PMID: 32796816. Available from: 10.1038/ s41419-020-02849-4.

2. Obara H, Matsubara K, Kitagawa Y. Acute limb ischemia. Ann Vasc Dis. 2018;11(4):443-448. PMID: 30636997. Available from: 10.3400/avd.ra.18-00074.

3. Olinic DM, Stanek A, Tataru DA, Homorodean C, Olinic M. Acute limb ischemia: an update on diagnosis and management. J Clin Med. 2019;8(8):1215. PMID: 31416204 . Available from: $10.3390 / \mathrm{jcm} 8081215$.

4. Vu NB, V NLT, Phi LT, Phan NK, Pham PV. Human menstrual blood-derived stem cell transplantation for acute hind limb ischemia treatment in mouse models. In Regenerative Medicine (pp. 205-215). Springer, London.;

5. Oladipupo S, Hu S, Kovalski J, Yao J, Santeford A, Sohn RE. VEGF is essential for hypoxia-inducible factor-mediated neovascularization but dispensable for endothelial sprouting. Proc Natl Acad Sci USA. 2011;108(32):13264-9. PMID: 21784979. Available from: 10.1073/pnas.1101321108.

6. Brenes RA, Jadlowiec CC, Bear M, Hashim P, Protack CD, Li X. Toward a mouse model of hind limb ischemia to test therapeutic angiogenesis. J Vasc Surg. 2012;56(6):1669-79. PMID: 22836102. Available from: 10.1016/j.jvs.2012.04.067.

7. Hu GW, Li Q, Niu X, Hu B, Liu J, Zhou SM. Exosomes secreted by human-induced pluripotent stem cell-derived mesenchymal stem cells attenuate limb ischemia by promoting angiogenesis in mice. Stem Cell Res Ther. 2015;6(1):10. PMID: 26268554. Available from: $10.1186 /$ scrt546.

8. Yin C, Liang Y, Zhang J, Ruan G, Li Z, Pang R, et al. Umbilical Cord-Derived Mesenchymal Stem Cells Relieve Hindlimb Ischemia through Enhancing Angiogenesis in Tree Shrews. Stem cells international. 2016;p. 9742034. Available from: $10.1155 / 2016 / 9742034$.

9. Jockenhoevel S, Zund G, Hoerstrup SP, Chalabi K, Sachweh JS, Demircan L, et al. Fibrin gel - advantages of a new scaffold in cardiovascular tissue engineering. Eur J Cardiothorac Surg. 2001;19(4):424-30. PMID: 11306307. Available from: 10.1016/ S1010-7940(01)00624-8.
10. Laurens N, Koolwijk P, de Maat MP. Fibrin structure and wound healing. J Thromb Haemost. 2006;4(5):932-9. PMID: 16689737. Available from: 10.1111/j.1538-7836.2006.01861.x.

11. Zisch AH, Schenk U, Schense JC, Sakiyama-Elbert SE, Hubbell JA. Covalently conjugated VEGF-fibrin matrices for endothelialization. J Control Release. 2001;72(1-3):101-13. PMID: 11389989. Available from: 10.1016/S0168-3659(01)00266-8.

12. Ceccarelli J, Putnam AJ. Sculpting the blank slate: how fibrin's support of vascularization can inspire biomaterial design. Acta Biomater. 2014;10(4):1515-23. PMID: 23933102. Available from: 10.1016/j.actbio.2013.07.043.

13. Dao TTT, Nguyen CTH, Vu NB, Le HTN, Nguyen PDN, Pham $P V$, et al. Evaluation of proliferation and osteogenic differentiation of human umbilical cord-derived mesenchymal stem cells in porous scaffolds. In Tissue Engineering and Regenerative Medicine. 2019;p. 207-220. Available from: 10.1007/ 5584_2019_343.

14. Pham PV, Truong NC, Le PT, Tran TD, Vu NB, Bui KH. Isolation and proliferation of umbilical cord tissue derived mesenchymal stem cells for clinical applications. Cell Tissue Bank. 2016;17(2):289-302. PMID: 26679929. Available from: 10 . 1007/s10561-015-9541-6.

15. Pham PV, Bich NV, Phan NK. Umbilical cord-derived stem cells (Modulatist TM) show strong immunomodulation capacity compared to adipose tissue-derived or bone marrow-derived mesenchymal stem cells. Biomed Res Ther. 2016;3(6):1-10. Available from: 10.7603/s40730-016-0029-1.

16. Dominici M, Blanc KL, Mueller I, Slaper-Cortenbach I, Marini F, Krause D. Minimal criteria for defining multipotent mesenchymal stromal cells. The International Society for Cellular Therapy position statement. Cytotherapy. 2006;8(4):315-7. PMID: 16923606. Available from: 10.1080/14653240600855905.

17. Pham PV. A comparison of umbilical cord blood-derived endothelial progenitor and mononuclear cell transplantation for the treatment of acute hind-limb ischemia. Biomed Res Ther. 2014;1(1):1-12. Available from: 10.7603/s40730-014-0001-x.

18. Vu NB, Phi LT, Dao TT, Le HT, Ta VT, Pham PV. Adipose derived stem cell transplantation is better than bone marrow mesenchymal stem cell transplantation in treating hind-limb ischemia in mice. Biomed Res Ther. 2016;3(9):46. Available from: 10.7603/s40730-016-0046-0.

19. Vu NB, Le HTN, Dao TTT, Phi LT, Phan NK. Allogeneic adiposederived mesenchymal stem cell transplantation enhances the expression of Angiogenic factors in a mouse acute Hindlimb ischemic model. In Stem Cells: Biology and Engineering 2017;p. 1-17. Available from: 10.1007/5584_2017_63.

20. Goto T, Fukuyama N, Aki A, Kanabuchi K, Kimura K, Taira H. Search for appropriate experimental methods to create stable hind-limb ischemia in mouse. Tokai J Exp Clin Med. 2006;31(3):128-32. PMID: 21302240.

21. Nie WB, Zhang D, Wang LS. Growth Factor Gene-Modified Mesenchymal Stem Cells in Tissue Regeneration. Drug Des Devel Ther. 2020;14:1241-56. PMID: 32273686. Available from: 10.2147/DDDT.S243944.

22. Liew A, O'Brien T. Therapeutic potential for mesenchymal stem cell transplantation in critical limb ischemia. Stem Cell Res Ther. 2012;3(4):28. PMID: 22846185. Available from: $10.1186 /$ scrt119.

23. Liao L. Cell therapy of critical limb ischemia: A review of preclinical and clinical research in China. Vasc Investig Ther. 2020;3(1):21. Available from: 10.4103/VIT.VIT_7_20.

24. Fan M. SymbolHuman umbilical cord mesenchymal stem cells transplantation improves lower limb ischemia of diabetic rabbits. J Clin Rehabil Tissue Eng Res. 2011;15(1):33-6.

25. Nguyen PD, Vu NB, Le HT, Dao TT, Gia LX, Pham PV. Engineered cartilage tissue from biodegradable Poly ( $\varepsilon$-caprolactone) scaffold and human umbilical cord derived mesenchymal stem cells. Biomed Res Ther. 2018;5(2):2000-12. Available from: 10.15419/bmrat.v5i02.414.

26. Oprea WE, Karp JM, Hosseini MM, Davies JE. Effect of platelet releasate on bone cell migration and recruitment in vitro. J 
Craniofac Surg. 2003;14(3):292-300. PMID: 12826799. Available from: 10.1097/00001665-200305000-00006.

27. Martín-Solé O, Rodó J, García-Aparicio L, Blanch J, Cusí V, Albert A. Effects of platelet-rich plasma (PRP) on a model of renal ischemia-reperfusion in rats. PLoS One. 2016;11(8):e0160703. PMID: 27551718. Available from: 10.1371/journal.pone. 0160703.

28. Jia Y, Qin J, Zhi Z, Wang RK. Ultrahigh sensitive optical microangiography reveals depth-resolved microcirculation and its longitudinal response to prolonged ischemic event within skeletal muscles in mice. J Biomed Opt. 2011;16(8):086004. PMID: 21895316. Available from: 10.1117/1.3606565.
29. Basbaum Al, Bautista DM, Scherrer G, Julius D. Cellular and molecular mechanisms of pain. Cell. 2009;139(2):267-84. PMID: 19837031. Available from: 10.1016/j.cell.2009.09.028.

30. Chen L, Tredget EE, Wu PY, Wu Y. Paracrine factors of mesenchymal stem cells recruit macrophages and endothelial lineage cells and enhance wound healing. PLoS One. 2008;3(4):e1886. PMID: 18382669. Available from: 10.1371/ journal.pone.0001886.

31. Hamada $Y$, Gonda K, Takeda M, Sato A, Watanabe M, Yambe T. In vivo imaging of the molecular distribution of the VEGF receptor during angiogenesis in a mouse model of ischemia. Blood. 2011;118(13):e93-100. PMID: 21821706. Available from: 10.1182/blood-2010-12-322842. 